\title{
PHYSICAL-BASED MODELS OF SPECKLE FOR HIGH RESOLUTION SAR IMAGES
}

\author{
Gerardo Di Martino, Antonio Iodice, Daniele Riccio, Giuseppe Ruello \\ Università di Napoli “Federico II”, Via Claudio 21, 80125 Napoli, Italy \\ \{gerardo.dimartino, iodice, daniele.riccio, ruello\}@unina.it
}

\section{INTRODUCTION}

During the last few years the new generation Synthetic Aperture Radar (SAR) sensors have been providing high resolution microwave images of the Earth. TerraSAR-X and COSMO-SkyMed are now able to produce data with a resolution of $1 \times 1 \mathrm{~m} 2$ in the spotlight operational mode: due to this increase in resolution, many of the models and techniques so far developed need to be updated to cope with this new scenario. When natural areas are observed, a key importance is assumed by the statistical description of the scene [1]: in fact, SAR images hold information on the long and short scale of the observed surface.

In the analysis of SAR data, the nature of the speckle phenomenon, due to the coherent character of the SAR sensor, must be carefully taken into account. In fact, the development of automatic or semi-automatic segmentation and classification techniques for the extraction of value-added products from these images is strongly dependent on an accurate modeling of the stochastic properties of the clutter, whose characteristics are in turn dependent on the sensor resolution.

The SAR signal measured in a resolution cell is typically modeled as the result of a superposition of electromagnetic contributions due to sub-pixel scatterers. Therefore, it can be seen as a single realization of a statistical process, that constitute the typical speckle noise [1].

The characteristics of speckle depend on the surface roughness, the view angle, the resolution, the synthetic antenna dimension in a non-linear way. Therefore, the speckle characterization has been so far faced with statistical models, dependent on the number of independent scatterers. For large number of scatterers, the speckle is considered fully developed, and the SAR signal, evaluated via the central limit theorem, exhibits uniform phase and Rayleigh distributed amplitude. If the number of scatterers does not allow the use of the central limit theorem, the speckle description requires different models, based on the number of scatterers as well as on the single scatterer description.

In literature, the problem is mainly approached in terms of statistical characterization, where the speckle is treated as a noise signal. Rayleigh [1] or K-distributed [2] noises are usually employed to describe the speckle 
characteristics as a function of the number of independent scatterers. In fact, the $\mathrm{K}$ distribution has been effectively used to model a wide range of clutter typologies (as an example, see [2]). The model proposed in [2] shows a parametric dependence on the number of independent scatterers in each resolution cell and, consequently, appears to be very well suited for the characterization of high resolution SAR clutter of extended scenes. Anyway, no relation between the number of scatterers and the physical properties of the observed surfaces can be found in the open literature.

In this paper, we present an analytical approach to the problem based on pure electromagnetic models. According to this approach, the speckle is no longer considered as a disturbing noise, but as a part of the signal, depending on the characteristics of the surface at scales lower than the resolution one. Natural surfaces are described by means of fractal functions [3]. The field scattered from the fractal surfaces is accounted for by means of the Kirchhoff approach (KA). In addition, we present a SAR raw signal simulator able to generate SAR images whose speckle noise is coherent with the observed surface properties.

\section{PHYSICAL MODELS}

The analytical method requires a reliable description of the observed surface and its interaction with the incident electromagnetic field. The fractal Weierstrass-Mandelbrot function is employed for the surface description [3]. Its use is chosen because it represents a predictable stochastic function, well suited to describe natural surfaces in terms of only two parameters, the fractal dimension $D$ and the standard deviation of increments at unitary distance $s[3]$.

The electromagnetic field scattered from the WM function is then evaluated in the frame of the KA. The obtained closed form solution is a superposition of modes, whose intensities and directions of propagation depend on the surface roughness, the facet dimension and the view angle [3]. A complete analysis in the backscattering case is able to provide the equivalent number $\mathrm{N}$ of scatterers as a function of these physical parameters. This model provides a meaningful relation between the physical surface properties (the fractal dimension and the standard deviation of the surface increments) and the equivalent number of scatterers, needed as input to speckle models.

The obtained results can be used as input for a SAR raw signal simulator, developed at the Department of biomedical, electronic, and telecommunications engineering [4]. In this paper, we extend the validity of this simulator, developed for simulating images with Rayleigh speckle, to the case of K-distributed speckle. 


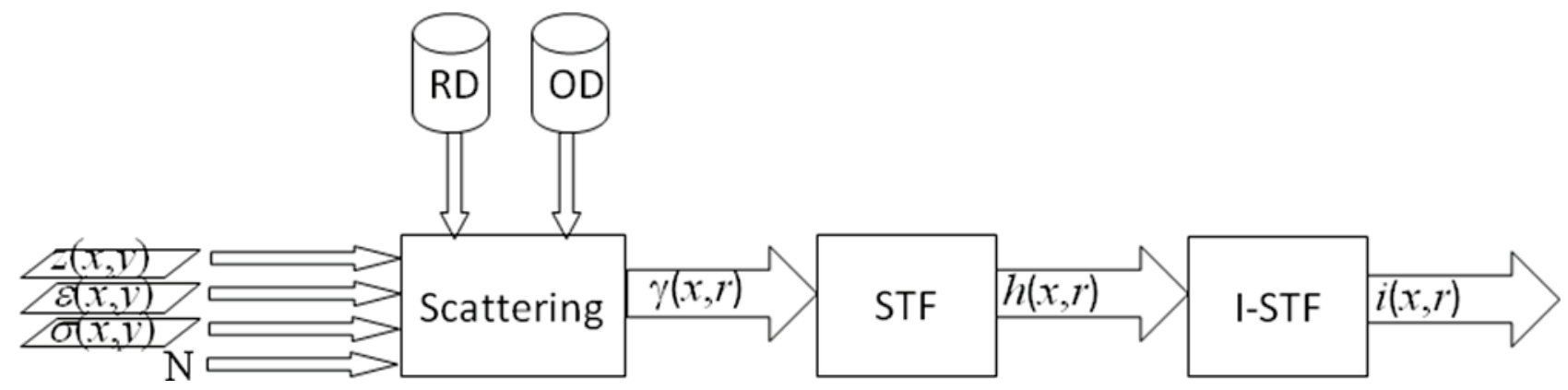

Fig.1 Block diagram of the simulation procedure

As shown in Figure 1, given the radar (RD) and orbital (OD) data and the fractal surface $\mathrm{z}(x, y)$, with its electromagnetic parameters $\varepsilon(x, y)$ and $\sigma(x, y)$, the mean scattering is evaluated via the KA and the speckle is accounted for as realization of K- or Rayleigh- distributed process. This leads to the reflectivity function $\chi(x, r)$, which is convolved with the SAR transfer function (STF) by means of a proper frequency domain elaboration in order to evaluate the raw signal $h(x, r)$. A standard processing is employed to retrieve the simulated image $i(x, r)$.

As a result of the presented procedure, we obtained a relation between the surface physical parameters and the speckle properties. A validation of the method will be provided by measuring the normalized moments of the simulated images with the theoretical moments relevant to Rayleigh, or K-distributed speckle. In the Rayleigh case, the n-order normalized intensity moments follow a $n$ ! law; for a K-distribution, the normalized moments are evaluated as:

$$
\frac{\left\langle W^{n}\right\rangle}{\langle W\rangle^{n}}=\frac{n !}{\alpha^{n}} \frac{\Gamma(n+\alpha)}{\Gamma(\alpha)},
$$

where $\Gamma$ is the Gamma function and $\alpha$ is related to the number of scatterers N. A parametric study on the effect of the physical surface properties on the simulated images will be provided in the final version of the paper.

\section{CONCLUSIONS}

In this paper a physical-based model of the SAR speckle noise is presented. It is based on the evaluation of the electromagnetic field scattered by a resolution cell. The natural surfaces are described in terms of fractal functions, and the scattered field is evaluated via the Kirchhoff approximation. This work intends to provide a powerful theoretical instrument for the interpretation of high resolution SAR images. 
In addition, the use of its results in the frame of a SAR raw signal simulator allowed the development of a new SAR simulator, able to deal with K-distributed speckle. Such a simulator provides a powerful instrument for comprehend SAR images and for training feature extraction algorithms.

\section{REFERENCES}

[1] Beckmann P., A. Spizzichino, Scattering of Electromagnetic Waves from Rough Surfaces, New York, NY: Artech House Publishers, 1987.

[2] Jakeman E. and P. N. Pusey, "A Model for Non-Rayleigh Sea Echo", Royal Signals and Radar Establishment, Malvern, Worcestershire, England, 1976..

[3] Franceschetti, G., D. Riccio, Scattering, Natural Surfaces and Fractals, Academic Press, Burlington, MA (USA), 2007.

[4] G. Franceschetti, M. Migliaccio, D. Riccio, G. Schirinzi, "SARAS: a SAR Raw Signal Simulator", IEEE Trans. Geosci. Remote Sens., vol. 30, no. 1, pp. 110-123, Jan.1992. 